\title{
Skin Barrier-enhancing, Antiwrinkle, and Antimelanogenic Effects of Probiotic Lysates Composed of Nucleotides
}

\author{
Kyung-Min Kim ${ }^{1}$, Ha-Yeon Kim ${ }^{1}$, So-Yoon Cha ${ }^{1}$, Ye-Hyang Kim ${ }^{1}$, Ji-Won Song ${ }^{1}$, and Seunghun Lee ${ }^{2, *}$ \\ ${ }^{1}$ Researcher, R\&D Center, HYUNDAI BIOLAND Co., Ltd. \\ ${ }^{2}$ Research Director, R\&D Center, HYUNDAI BIOLAND Co., Ltd.

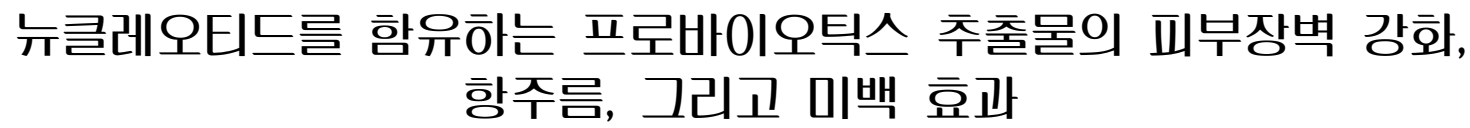 \\ 김경민 ${ }^{1} \cdot$ 김하연 ${ }^{1} \cdot$ 차소윤 $^{1} \cdot$ 김예향 ${ }^{1} \cdot$ 송지원 ${ }^{1} \cdot$ 이승훈 ${ }^{2, *}$ \\ ${ }^{1}$ (주)현대바이오랜드, 연구원 \\ 2(주)현대바이오랜드, 연구소장
}

\begin{abstract}
Several previous studies have investigated the skin aging prevention effects of ceramide, hyaluronic acid, and natural or fermented plant materials. Recently, oral administration and dermal application of probiotics or probiotic lysates have shown antiaging effects. The purpose of this study is to optimize the preparation of probiotic lysates with a high concentration of nucleotides and to confirm the effects of probiotic lysates on the skin. Probiotic lysates were prepared by heating at $121^{\circ} \mathrm{C}$ for various periods with adding of sodium hyaluronic acid. Probiotic lysates of Bifidobacterium longum HDB7072, Lactobacillus paracasei HDB1196, and Lactobacillus acidophilus HDB1014 were applied to normal human epidermal keratinocytes (NHEKs), fibroblast cells, and B16F1 cells, respectively. Cell viability, antioxidant effects, and mRNA expression were evaluated by using MTT assays, DPPH assays, and qRT-PCR. Probiotic lysates prepared by heating the culture medium at $121^{\circ} \mathrm{C}$ for $2 \mathrm{~h}$ with $0.5 \%$ sodium hyaluronic acid showed the highest nucleotide concentration. In the three tested skin cells, the cell viability of filtered lysates was similar or higher to that of unfiltered lysates. HDB7072 lysates increased filaggrin expression in NHEKs. HDB1196 lysates showed DPPH radical-scavenging and antiwrinkle effects through the downregulation of matrix metalloproteinase- 1 and upregulation of collagen type 1 in fibroblasts. HDB1014 lysates had antioxidant and antimelanogenic effects in $\mathrm{B} 16 \mathrm{~F} 1$ cells. Cell wall-removed probiotic lysates could be used as novel ingredients to improve skin aging and skin barrier issues.
\end{abstract}

Keywords: Antimelanogenic, Antiwrinkle, Probiotics, Skin aging, Skin barrier

\section{Introduction}

The skin is the largest sensory organ in the human body and consists of three layers; the epidermis, dermis, and subcutaneous fat layer. Skin protects the human body from the external environment, synthesizes vitamin $\mathrm{D}$, prevents water loss, and maintains a constant body temperature (Jung et al., 2019). As the skin ages due to age, diet, and environmental factors such as ultraviolet (UV) radiation, chemical

*Corresponding author: Seunghun Lee

Tel : +82-31-8085-7514

E-mail : shunlee@hyundaibioland.co.kr

접수일(2021년 8월 25일)/수정일(2021년 10월 25일)/채택일(2021년 11월 29일) substances, and fine particles, skin structures begin to function abnormally (Krutmann et al., 2017). The most common symptoms of skin aging are skin barrier weakness and the formation of wrinkles, melasma, and freckles (Puisina-Ivuc, 2008).

Previous research on protection from skin aging has focused largely on the effects of natural plant and animal materials or their fermented products. Recently, however, microorganisms have also been investigated for their ability to suppress and slow down skin aging. Lactic acid bacteria (LAB) are representative probiotic strains that can provide health-promoting effects such as antiobesity, antiinflammation, antioxidant, and cholesterol-lowering effects (Kim et al., 
2015). In addition to intestinal benefits, recent studies have reported that $\mathrm{LAB}$ and their extracts can improve skin conditions and prevent skin problems. Applying LAB and their lysates directly to the skin can improve skin conditions. For instance, Lactobacillus rhamnosus lysate crushed with a microfluidizer improved the skin barrier function in reconstructed human epidermis (Jung et al., 2019). Additionally, Bifidobacterium longum and Lactobacillus spp. lysed by a bead beater induced the upregulation of tight junction barrier genes in human keratinocytes (Sultana et al., 2013). The extract of Lactobacillus paracasei and L. rhamnosus showed antiwrinkle activities by decreasing production of matrixmetalloproteinase-1 (Kim et al., 2015). Furthermore, heatkilled Lactobacillus acidophilus KCCM12625P was able to regulate wrinkle-related and melanogenesis-related genes regulation in UVB-irradiated cells (Lim et al., 2020). Finally, Lactobacillus reuteri DSM 17938 lysed by a mechanical high-pressure homogenizer caused antiinflammatory and hydration effects in reconstructed human epidermis (Khmaladze et al., 2019).

Although several probiotic lysates prepared by various means have been evaluated, their indicators and functional substances have yet to be clarified. In addition, for most probiotic lysates, the whole component, including culture media and cell wall, has typically been applied after cells were crushed. Some researchers have reported that lipopolysaccharides (LPS) from the cell wall and lactic acid in culture media could irritate the skin (Aramaki et al., 2002; Cruz et al., 2001). Therefore, the objective of this study is to identify the functional substances of probiotic lysate and optimize a lysate preparation method to include a high concentration of nucleotides and remove cells walls and culture media. Probiotic lysates were prepared using an optimized method with species previously reported to be effective, namely, L. acidophilus, L. paracasei, and $B$. longum, and the skin effects of these probiotic lysates were compared.

\section{Materials and Methods}

\section{Bacterial strains and cell culture}

L. acidophilus HDB1014, L. paracasei HDB1196, and B. longum HDB7072 were provided by Cell Bank (HYUNDAI BIOLAND Co., Ltd., Korea). Lactobacillus strains were grown in de Man, Rogosa, and Sharpe (MRS; BD BBL, Franklin Lakes, NJ, USA) broth, and the Bifidobacterium strain was grown in BL (MB-B1602, KisanBio Co., Ltd., Korea) broth at $37^{\circ} \mathrm{C}$ for $24 \mathrm{~h}$.

Human dermal fibroblasts were obtained from American Type Culture Collection (ATCC; USA) and cultured using Iscove's modified Dulbecco's medium (WELGENE, Korea). B16F1 cells (mouse melanoma cells) were purchased from Korean Cell Line Bank (KCLB; Korea) and cultured using Dulbecco's Modified Eagle Medium (WELGENE). These cells were cultured with 10\% fetal bovine serum (Gibco, USA) and $1 \%(\mathrm{v} / \mathrm{v})$ streptomycin/penicillin solution in an incubator with $5 \% \mathrm{CO}_{2}$ at $37^{\circ} \mathrm{C}$. Normal human epidermal keratinocytes (NHEKs) were obtained from Cascade Biologics, Inc. (USA), and cultured in Epilife medium (Cascade Biologics) supplemented with human keratinocyte growth supplement. NHEKs were grown in culture dishes in an incubator with 5\% $\mathrm{CO}_{2}$ at $37^{\circ} \mathrm{C}$, and the culture medium was replaced every 2 days.

\section{Optimization of probiotic lysates preparation}

All probiotic strains were grown in MRS or BL broth at $37^{\circ} \mathrm{C}$ for $24 \mathrm{~h}$. Each overnight culture was adjusted to approximately $10^{8} \mathrm{CFU} / \mathrm{mL}$, centrifuged at $5,782 \times g$ for 10 min, and then washed three times with phosphate-buffered saline. The harvested cells were inoculated into distilled water or sodium hyaluronate solutions $[0.25 \%, 0.50 \%$, and $1 \%$ (w/ v) sodium hyaluronate in distilled water] and incubated at $37^{\circ} \mathrm{C}$ for $24 \mathrm{~h}$. To produce probiotic lysates, the overnight cultures were heated at $121^{\circ} \mathrm{C}$ for $15,30,60,120$, and 150 min. After filtration with a $0.45 \mu \mathrm{m}$ filter to remove cell walls, the probiotic lysates were completed. The nucleotide concentration, i.e., the active components of probiotic lysates, were measured using a NANODROP 2000 spectrophotometer (Thermo Fisher Scientific, Waltham, MA, USA).

To determine the necessity of filtration, the cell cytotoxicity of filtered and unfiltered probiotic lysates was measured using a 3-(4,5-dimethylthiazol-2-yl)-2,5-diphenyl tetrazolium bromide (MTT; Promega, USA) assay (Kim et al., 2020). The probiotic lysates were applied to each well of 24-well plates containing the three tested skin cells and incubated at $37^{\circ} \mathrm{C}$ for $24 \mathrm{~h}$. After the supernatant was removed, MTT solution $(0.25 \mathrm{mg} / \mathrm{mL})$ was applied to each well at $37^{\circ} \mathrm{C}$ for $4 \mathrm{~h}$ before DMSO was added to the wells. The absorbance of the 
mixtures was then measured at $570 \mathrm{~nm}$.

\section{Radical-scavenging activity assay}

The radical-scavenging activity of probiotic lysates was measured using a DPPH (2,2-diphenyl-1-picrylhydrazyl) assay (Kim et al., 2015). Distilled water and $0.1 \mathrm{mg} / \mathrm{mL}$ ascorbic acid (Vit C; Sigma Aldrich, USA) were used as control groups. Samples $(150 \mu \mathrm{L})$ were mixed with the same amount of DPPH solution ( $0.1 \mathrm{mM}$ in methanol). The mixtures were then incubated at room temperature for $30 \mathrm{~min}$ in the dark, and then the absorbance of the mixtures was measured at $517 \mathrm{~nm}$.

\section{Skin barrier enhancement assay}

To evaluate fillaggrin (FLG) expression, NHEKs were inoculated and incubated for $24 \mathrm{~h}$. After the culture media were removed, ceramide NP (Evonik Degussa, Germany, 100 $\mu \mathrm{g} / \mathrm{mL}$ ), as a positive control for keratinocyte differentiation, or B. longum HDB7072 lysate (HDB7072) were treated to NHEKs and then incubated for $24 \mathrm{~h}$.

\section{Antiwrinkle assay}

To evaluate the inhibition of matrix metalloproteinase-1 (MMP-1) gene expression, fibroblast cells were inoculated and incubated for $24 \mathrm{~h}$. Cells were irradiated with $6 \mathrm{~J} / \mathrm{cm}^{2}$ UVA and incubated for $24 \mathrm{~h}$ with $2.5 \mu \mathrm{g} / \mathrm{mL}$ epigallocatechin gallate (EGCG; Sigma Aldrich) and L. paracasei HDB1196 lysate (HDB1196). To evaluate collagen type 1 (COL-1) gene expression, fibroblast cells were inoculated and incubated for 24 h. Subsequently, $75 \mu \mathrm{g} / \mathrm{mL}$ Vit C or HDB1196 was inoculated into each well and incubated for $24 \mathrm{~h}$. EGCG and Vit $\mathrm{C}$ were used as positive controls.

\section{Antimelanogenic assay}

B16F1 cells were inoculated and incubated at $37^{\circ} \mathrm{C}$ for 24 h. The culture media were changed to media with $100 \mathrm{nM} \alpha-$ melanocyte stimulating hormone ( $\alpha$-MSH; Sigma Aldrich) to stimulate melanogenesis. L. acidophilus HDB1014 lysate (HDB1014) was then applied, and cells were incubated for 72 h. To evaluate the melanin secretion rate, the absorbance of the culture medium was measured at $450 \mathrm{~nm}$. To evaluate melanin content, cells were dissolved with $250 \mu \mathrm{L}$ of $1 \mathrm{~N}$ $\mathrm{NaOH}$ at $50^{\circ} \mathrm{C}$ for $15 \mathrm{~min}$. The absorbance of the mixtures was measured at $450 \mathrm{~nm}$. Arbutin was used as a positive control.

\section{Quantitative real-time polymerase chain reaction (qRT-PCR)}

After cells were cultured with the samples for each experiment, total mRNA was extracted using QIAzol Lysis Reagent (Qiagen, Hilden, Germany) according to the manufacturer's instruction. Subsequently, mRNA concentrations were measured using a Qubit RNA BR Assay Kit (Invitrogen, USA), and cDNA was synthesized using a qPCRBIO cDNA Synthesis Kit (PCR Biosystems, London, UK). To quantify the expression of the barrier gene (FLG) and wrinkle gene (MMP-1 and COL-1), the primers shown in Table 1 were used. A real-time PCR kit (qPCRBIO SyGreen Blue Mix Lo-ROX, PCR Biosystems, BioD Co., Ltd., Korea) was used to perform aRTPCR on a 7300 Real-Time PCR system (Applied Biosystems, USA).

\section{Statistics}

All results are shown as the mean \pm standard deviation (SD) of at least three experiments. The control and experi-

Table 1. Primer sequences used for qRT-PCR

\begin{tabular}{cll}
\hline Target & Orientation & \multicolumn{1}{c}{ Sequence $\left(5^{\prime}\right.$ to $\left.3^{\prime}\right)$} \\
\hline Filaggrin (FLG) & Forward & CACGTGGCAGTCCTCACAGT \\
& Reverse & CTTTTTGCCTTCAGTGCCC \\
Matrix metalloproteinase-1 (MMP-1) & Forward & GTTCAGGGACAGAATGTGCTACAC \\
& Reverse & TCTAGGGAAGCCAAAGGAGCT \\
Collagen type 1 (COL-1) & Forward & AGCAAGAACCCCAAGGACAA \\
& Reverse & CGAACTGGAATCCATCGGTC \\
\multirow{2}{*}{$\beta$-Actin } & Forward & GGCACCCAGCACAATGAAG \\
& Reverse & CCGATCCACACGGAGTACTTG
\end{tabular}


mental groups were compared using Mann-Whitney U test in SPSS Static 19.0 (SPSS, Inc., Chicago, IL, USA).

\section{Results and Discussion}

\section{Optimization of probiotic lysate manufacturing}

Studies on the skin health effects of probiotics and their cultures are actively being conducted, and lipoteichoic acid (LTA), phospholipids in cell membranes, oligosaccharides, short chain fatty acids, and peptides are known to be functional substances (Kim \& Lee, 2015). However, using the entire bacteria and culture may have disadvantages as it may contain harmful substances. Thus, it is important to purify functional substances that can easily to be analyzed and quantified.

Nucleotides are the units of DNA and RNA, which are natural polymers and play a pivotal role in metabolism in living organisms. Many researchers have studied the functionality of nucleotides or their components such as adenosine. Salmon milt DNA has been used for medical therapy and is reported to have wound healing, antiinflammatory, and UV protection effects on the skin (Nakamichi et al., 2019; Sasaki et al., 2010; Sato et al., 2017; Shen et al., 2008). Besides salmon milt DNA, bacterial genomic DNA of heat-killed $L$. plantarum has been shown to have antiinflammatory effects through the secretion of elafin on Caco-2 cells mediated by toll-like receptor 9 (TLR9) (Hiramatsu et al., 2019). In addition, adenosine is known to have wound healing, antiinflammatory, and dermal collagen production effects (Cronstein, 2011). Collectively, these results of research indicate that the nucleotides of LAB could be substances that promote skin health.

To increase the nucleotide concentration of probiotic lysates, the heating time and addition of sodium hyaluronate were varied in the present study. Until $2 \mathrm{~h}$ of heating and $0.5 \%$ of sodium hyaluronate, the concentration of nucleotides increased as heating time and sodium hyaluronate concentration increased (Fig. 1A). Specifically, probiotic lysate heated at $121^{\circ} \mathrm{C}$ for $2 \mathrm{~h}$ with $0.5 \%(\mathrm{w} / \mathrm{v})$ sodium hyaluronate had the highest nucleotide content of $268.51 \mathrm{ng} / \mu \mathrm{L}$. It is presumed that nucleotide content increased with heating time due to the continued disruption of the cell wall of probiotics. At the second fermentation step, sodium hyaluronate likely increased nucleotide content because, when used as a nutrient, it promotes LAB growth. In previous studies, the growth of LAB such as L. acidophilus, L. rhamnosus, and Streptococcus thermophilus was enhanced in MRS broth with $0.03 \%-0.10 \%$
A
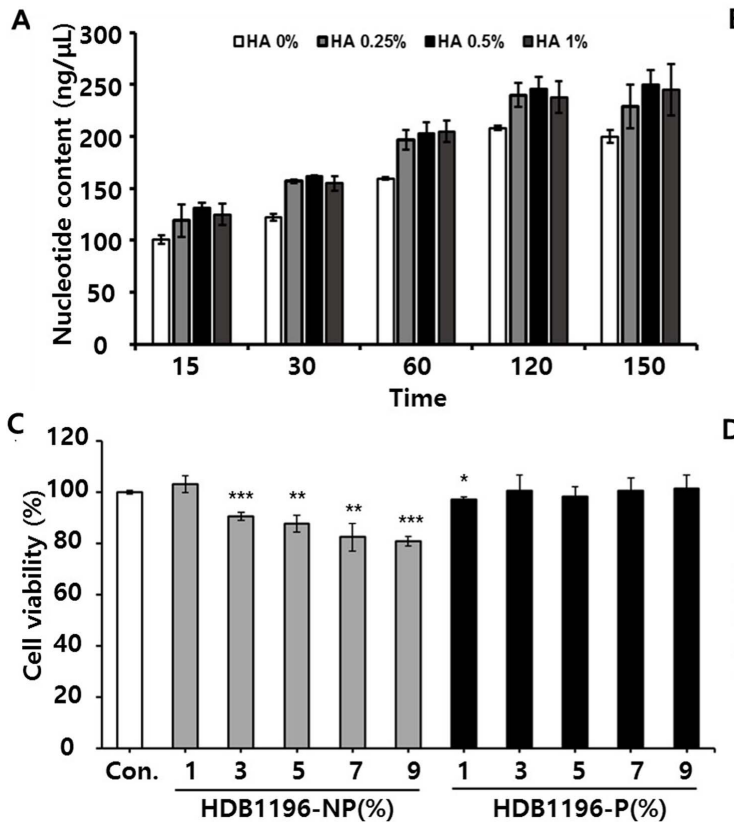

B
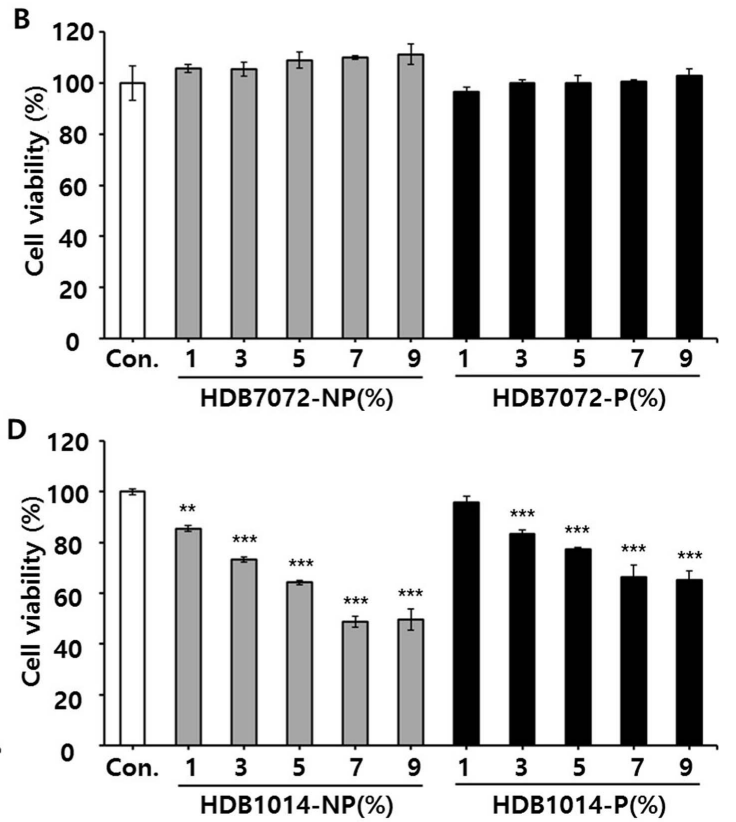

Fig. 1. The nucleotide concentration and cell viability of probiotic lysates. (A) The nucleotide concentration of $L$. paracasei HDB1196 lysates. Cell viability of unfiltered (NF) and filtered (F) probiotic lysates in normal human epidermal keratinocytes (B: treated with B. longum HDB7072 lysate), fibroblasts (C: treated with L. paracasei HDB1196 lysate), and B16F1 cells (D: treated with L. acidophilus HDB1014 lysate). Data are means \pm SDs of three independent experiments. Different letters on bars indicate significant differences (at $p<0.05)$ among values: ${ }^{*} p<0.05,{ }^{* *} p<0.01$, and ${ }^{* * *} p<0.001$ vs. control. 


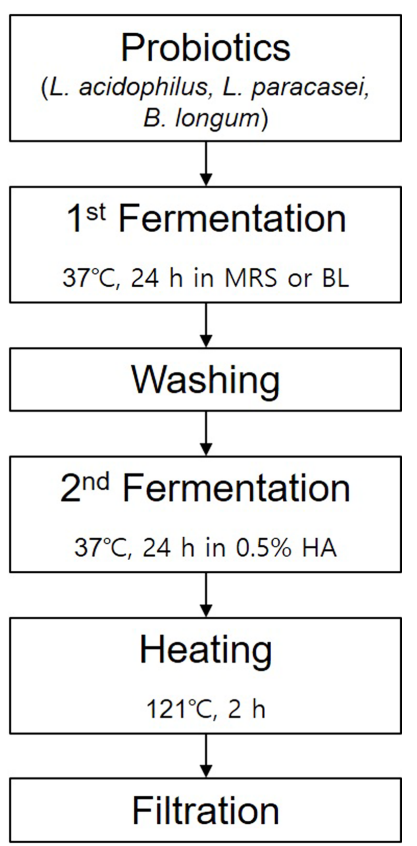

Fig. 2. Production process of probiotic lysates. HA, sodium hyaluronic acid.

sodium hyaluronate in a dose-dependent manner (Cong Nguyen, 2010; Di Cerbo et al., 2013).

The MTT assay showed that filtration was a necessary process for reducing skin irritation on skin cells. Although the cytotoxicity of unfiltered and filtered HDB7072 treatments was not significantly different to that of untreated NHEKs (control) at concentration up to $9 \%$ (v/v) (Fig. 1B). However, filtered HDB1196 and HDB1014 treatments were less cytotoxic than unfiltered lysates in B16F1 and fibroblast cells. Filtered HDB1196 did not affect viability at concentrations up to $9 \%(\mathrm{v} / \mathrm{v})$, whereas unfiltered lysates affected viability at 9\% (v/v) (Fig. 1C). In addition, filtered HDB1014 had no effect viability at concentrations up to $3 \%(\mathrm{v} / \mathrm{v})$, whereas unfiltered lysates affected cell viability at $3 \%$ (v/v) (Fig. 1D). Considering these data, the probiotic lysates used in the subsequent experiments were prepared by first heating probiotics cells at $121^{\circ} \mathrm{C}$ for $2 \mathrm{~h}$ with $0.5 \%(\mathrm{w} / \mathrm{v})$ sodium hyaluronate and then by filtering (Fig. 2). In the subsequent functional evaluation, samples were treated at a maximum of $3 \%$ according to the cell safety concentration of the HDB1014 strain.

\section{Skin barrier-enhancing effects of $B$. longum HDB7072 lysates on NHEKs}

The stratum corneum is composed of a "brick and mortar" structure wherein proteins form the brick (e.g., keratin

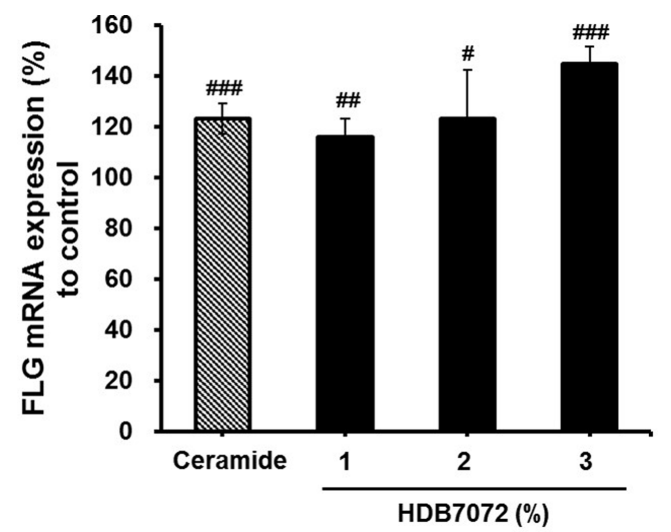

Fig. 3. Filaggrin (FLG) expression following B. longum HDB7072 lysate (HDB7072) treatment in normal human epidermal keratinocytes (NHEKs). NHEKs were treated with $100 \mu \mathrm{g} / \mathrm{mL}$ ceramide NP or HDB7072. Data are means \pm SDs of three independent experiments. ${ }^{\#} p<0.05,{ }^{\#} p<0.01$, and ${ }^{\# \#} p<0.001$ vs. control.

microfibrils and filaggrin) and lipids form the mortar (e.g., ceramides, free fatty acids, and cholosterol) (Jung et al., 2019). The skin barrier on which this theory is based is important not only for protection from the outside but also for protection of water loss from the skin. Skin barrier dysfunction, which causes conditions such as atopic dermatitis, psoriasis, and ichthyosis, is related to mutant filaggrin gene (flg), tight junction disorder, and reductions in ceramides, serine protease inhibitors, and antibacterial peptides (Bäsler et al., 2016).

The mRNA expression of FLG was increased in the HDB7072-treated cells in a dose-dependent manner (Fig. 3). The FLG expression of ceramide-treated and $3 \%(\mathrm{v} / \mathrm{v})$ HDB7072-treated cells increased by $123.1 \%$ and $144.5 \%$ compared with $100 \%$ of the control (distilled water), respectively. Thus, HDB7072 was shown to have a skin barrierimproving effect.

In previous research, the skin barrier-improving effects of other probiotic lysates have been reported. For instance, the microfluidized lysate of L. rhamnosus was shown to improve the expression of tight junction proteins [claudin (CLDN)-1, occludin (OCC)] and skin barrier proteins (FLG and loricrin) in a reconstructed human epidermis (Jung et al., 2019). In addition, L. rhamnosus and B. longum lysates, lysated with a bead beater, increased transepithelial electrical resistance and expression of tight junction proteins such as CLDN-1, CLDN-4, OCC, and ZO-1 (Sultana et al., 2013).

\section{Antiwrinkle effects of $L$. paracasei HDB1196 lysates on fibroblast cells}




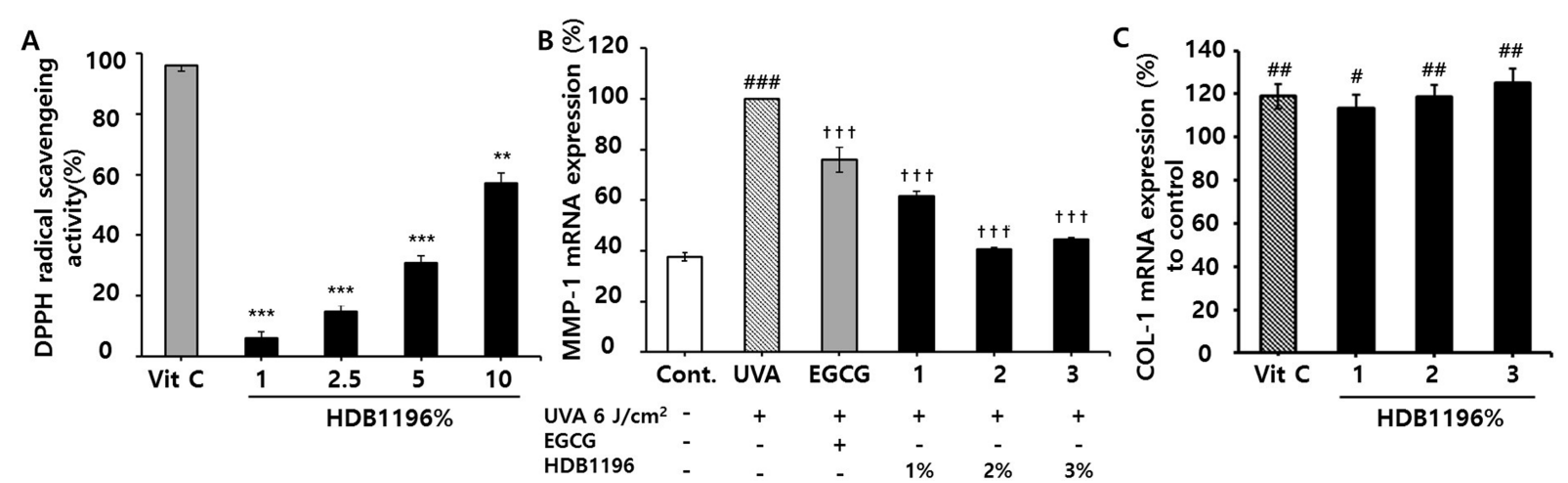

Fig. 4. Antioxidant and antiwrinkle effects of $L$. paracasei HDB1196 lysate (HDB1196) on fibroblast cells. (A) DPPH radical-scavenging activity (\%). (B) Fibroblast cells were irradiated with $6 \mathrm{~J} / \mathrm{cm}^{2}$ and then treated with EGCG $(2.5 \mu \mathrm{g} / \mathrm{mL}$ epigallocatechin) or HDB1196. Matrix metalloproteinase-1 (MMP-1) expression was measured using qRT-PCR. (C) Fibroblast cells were treated with Vit C (75 $\mu \mathrm{g} / \mathrm{mL}$ ascorbic acid) or HDB1196. Collagen type 1 (COL-1) expression was measured using qRT-PCR. Data are means \pm SDs of three independent experiments. ${ }^{* *} p$ $<0.01$, and ${ }^{* * *} p<0.001$ vs. Vit C. ${ }^{\#} p<0.05,{ }^{\# \#} p<0.01$, and ${ }^{\# \#} p<0.001$ vs. control; ${ }^{\dagger} p<0.05,{ }^{\dagger \dagger} p<0.01$, and ${ }^{\dagger \dagger} p<0.001$ vs. UVA group.

Wrinkles are the most common symptom of aging skin. Collagen and elastin are the main components of the extracellular matrix in the dermis, and these give the skin elasticity. Therefore, inhibiting the loss of collagen and elastin is an important factor in preventing skin aging. In particular, collagen production and MMP expression and activity are the most important factors in wrinkle formation (Park et al., 2019). Reactive oxygen species (ROS) accelerate the skin aging by promoting the degradation of collagen and the production of MMP-1, which is an interstitial collagenase that breaks down collagen fibers (Hong et al., 2015).

HDB1196 showed DPPH radical-scavenging activity in a dose-dependent manner (Fig. 4A). The mRNA expression of MMP-1 was markedly increased in the UVA-irradiated cells (Fig. 4B). Compared with the expression in the UV-irradiated cells, HDB1196 reduced MMP-1 expression levels by about $60 \%$, whereas EGCG reduced these levels by $24 \%$. By contrast, 3\% (v/v) HDB1196-treated and Vit C-treated cells showed increased COL-1 mRNA expression (124.9\% and $118.9 \%$, respectively) compared with $100 \%$ of untreated cells (Fig. 5C). Furthermore, the antioxidant effect of HDB1196 seemed to suppress wrinkle formation by counteracting ROS and decreasing MMP-1 levels.

In previous studies, the antiwrinkle effects of other probiotic lysates have been reported. In one study, lysates of $L$. paracasei and L. rhamnosus manufactured using an ultrasonic shredder showed DPPH radical-scavenging activity, upregulation of collagen, and downregulation of MMP-1 (Kim \& Lee, 2015). In another study, heat-killed L. acidophilus KCCM12625P showed antioxidant effects, increased levels of COL-1, and inhibition of elastase and MMP expression, which occurred through the inhibition of the phosphorylation of extracellular signal-regulated kinases (ERKs) and c-Fos in the activator protein-1 (AP-1) pathway (Lim et al., 2020).
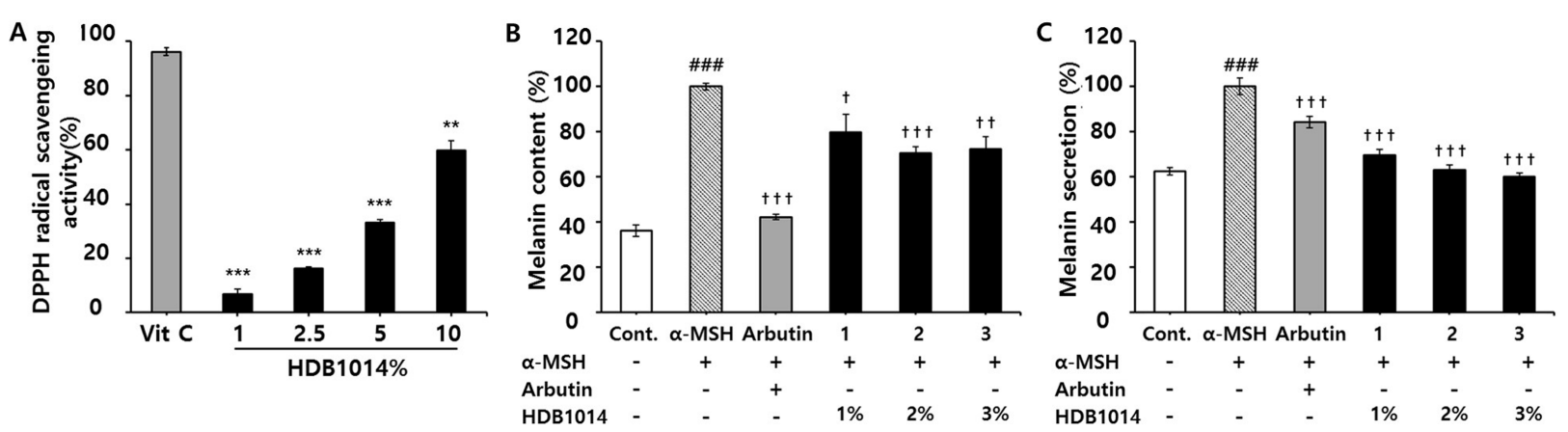

Fig. 5. The antioxidant and antimelanogenic effects of L. acidophilus HDB1014 lysate (HDB1014) on $\alpha$-melanocyte stimulating hormone ( $\alpha$ MSH)-induced B16F1 cells. (A) DPPH radical-scavenging activity (\%). B16F1 cells were treated with $100 \mathrm{nM} \alpha-\mathrm{MSH}$ and $100 \mu \mathrm{g} / \mathrm{mL}$ arbutin or HDB1014 for 3 days. (B) Melanin content (\%) of B16F1 cells lysis buffer. (C) Melanin secretion (\%) from the culture medium. Data are means \pm SDs of three independent experiments. ${ }^{* *} p<0.01$, and ${ }^{* * *} p<0.001$ vs. Vit C. ${ }^{\# \#} p<0.001$ vs. control; ${ }^{\dagger} p<0.05,{ }^{\dagger} p<0.01$, and ${ }^{\dagger \dagger \dagger} p<$ 0.001 vs. $\alpha$-MSH group. 
4. Inhibition of melanogenesis by $L$. acidophilus HDB1014 lysates in $\mathrm{B} 16 \mathrm{~F} 1$ cells

Melanogenesis is the process by which melanin is produced by melanocytes in melanosomes. Melanin, which is related to the color of the skin, hair, and eyes, protects the skin from UV radiation (Jang et al., 2020). However, oversynthesis and accumulation of melanin lead to the formation of melasma and freckles on the skin (Park et al., 2019). Tyrosinase is involved in the melanogenesis pathway, and it degrades tyrosine into L-3,4-dihydroxyphenylalanine (L-DOPA), which is, in turn, converted to dopachrome and melanin via an oxidation reaction with ROS (Kim \& Lee, 2015). Thus, antioxidant effects are related to antimelanogenic effects.

HDB1014 showed DPPH radical-scavenging activity in a dose-dependent manner (Fig. 5A). Melanin content and secretion were noticeably increased in $\alpha$-MSH-induced cells compared with levels in untreated cells. HDB1014 treatment inhibited melanin content in the cells and secretion from the cells. Melanin content and secretion in arbutin-treated cells were reduced by $57.8 \%$ and $15.7 \%$, respectively, whereas HDB1014 at 3\% (v/v) inhibited melanin production by $27.6 \%$ and secretion by $40 \%$ compared with the levels in $\alpha-\mathrm{MSH}-$ induced cells (Fig. 5B, 5C). Moreover, the antioxidant effect of HDB1014 seemed to suppress the oxidation reaction of melanogenesis by removing ROS and thereby reduced melanin content in $\mathrm{B} 16 \mathrm{~F} 1$ cells.

The antimelanogenetic effect of other probiotic lysates has previously been reported. For example, tyndallized $L$. acidophilus KCCM12625P showed ABTS radical-scavenging activity and antimelanogenic effects through the regulation of the cyclic adenosine monophosphate (cAMP) signaling-mediated pathway elements such as protein kinase A (PKA), cAMP response element binding (CREB), microphthalmia-associated transcription factor (MITF), and tyrosinase family members (Lim et al., 2020). In addition, L. paracasei and L. rhamnosus lysates showed DPPH radical-scavenging activity and the ability to reduce melanin content (Kim \& Lee, 2015).

Researchers have reported that LTA, a major component of the cell wall of gram-positive bacteria, has antioxidant, antiwrinkle, and antimelanogenic effects. LTA of $L$. plantarum and $L$. sakei has been shown to produce antioxidant effects and inhibit MMP-1 expression in UV-induced cells by inhibiting AP-1 and nuclear factor kappa-light-chain-enhancer of activated B cell (NF- $\mathrm{\kappa B}$ ) signaling pathways (Hong et al.,
2015; You et al., 2013). In addition, LTA isolated from $L$. plantarum is reported to produce antimelanogenic effects through the activation of ERK and the phosphatidylinositol 3kinase (PI3K)/AKT signaling pathway, e.g., via elements such as ERK, PI3K p85a, PI3K p110 $\beta$, AKT, MITF, and tyrosinase family members (Kim et al., 2015). In this study, probiotic samples were filtered, so the cell wall, including LTA, was largely removed. However, these probiotic lysates exerted the same skin functionality effects as lysates with intact cells. Therefore, in addition to the cell wall, nucleotides can be considered to act as functional substances. In conclusion, the probiotic lysates studied here could be potential ingredients for treatments of specific skin aging.

\section{Conclusion}

Skin aging is one of the important concerns of modern people, which is accompanied by symptoms such as collapse of the skin barrier, wrinkles, and freckles depending on internal and external factors. In this study, probiotic lysate from which cell wall components have been removed, each effective in skin barrier enhancing, antiwrinkle, and whitening, were developed as skin antiaging cosmetic materials. Three types of probiotic lysates (B. longum HDB7072, L. paracasei HDB1196, and L. acidophilus HDB1014) were prepared by extracting the nucleotide content, expected as an active material, through post-fermentation process with $0.5 \%$ sodium hyaluronate. HDB7072 significantly increased the gene expression of FLG, a skin barrier protein, in the NHEKs. HDB1196 significantly reduced collagen reduction and MMP-1 enzyme expression by UV treatment in the fibroblast. HDB1014 decreased the amount of melanin synthesis and secretion, which is an indicator of MSH-induced melengenesis in the B16F1 cells. Through these results, it was confirmed that three types of cell wall-removed probiotic lysates (HDB7072, HDB1196, and HDB1014) showed efficacy for skin barrier enhancing, antiwrinkle, and whitening, respectively, as a function for the development of antiaging cosmetic materials.

\section{References}

Aramaki, J., Kawana, S., Effendy, I., Happle, R., \& Loffler, H. (2002). Differences of skin irritation between Japanese and European women. British Journal of Dermatology, 146, 10521056. doi: 10.1046/j.1365-2133.2002.04509.x 
Bäsler, K., Bergmann, S., Heisig, M., Naegel, A., Zorn-Kruppa, M., \& Brandner, J. M. (2016). The role of tight junctions in skin barrier function and dermal absorption. Journal of Controlled Release, 242, 105-118. doi: 10.1016/j.jconrel.2016.08.007

Cong Nguyen, C. (2010). The effects of hyaluronic acid on the morphological physiological differentiation of Lactobacillus. Journal of Chemical and Pharmaceutical Research, 8, 368-372. doi: 10.8.20.7:8080/xmlui/handle/123456789/288

Cronstein, B. N. (2011). Adenosine receptors and fibrosis: a translational review. F1000 Biology Reports, 3, 21. doi: 10.3410/B3-21

Cruz, M. T., Duarte, C. B., Gonçalo, M., Carvalho, A. P., \& Lopes, M. C. (2001). LPS induction of IkB- $\alpha$ degradation and iNOS expression in a skin dendritic cell line is prevented by the janus kinase 2 inhibitor, tyrphostin B42. Nitric Oxide, 5, 53-61. doi: 10.1006/niox.2000.0320

Di Cerbo, A., Aponte, M., Esposito, R., Bondi, M., \& Palmieri, B. (2013). Comparison of the effects of hyaluronidase and hyaluronic acid on probiotics growth. BMC Microbiology, 13, 243. doi: 10.1186/1471-2180-13-243

Hiramatsu, Y., Sakamoto, D., Satho, T., Irie, K., Miake, F., Maeda, M., \& Kashige, N. (2019). Lactobacillus plantarum induces genomic DNA-dependent and TLR9-mediated elafin secretion from Caco-2 cells. Asian Pacific Journal of Allergy and Immunology, 37, 36-42. doi: 10.12932/AP-021017-0174

Hong, Y. F., Lee, H. Y., Jung, B. J., Jang, S., Chung, D. K., \& Kim, H. (2015). Lipoteichoic acid isolated from Lactobacillus plantarum down-regulates UV-induced MMP-1 expression and upregulates type I procollagen through the inhibition of reactive oxygen species generation. Molecular Immunology, 67, 248-255. doi: 10.1016/j.molimm.2015.05.019

Jang, D. K., Jung, S. H., Jeong, J. H., Yoo, H. M., Lee, I. S., \& Shin, H. S. (2020). The antimelanogenic effect of Inularin isolated from flowers of Inula britannica on B16F10 melanoma cells and zebrafish embryos. Journal of Microbiology and Biotechnology, 30, 749-752. doi: 10.4014/jmb.2003.03025

Jeong, J. H., Lee, C. Y., \& Chung, D. K. (2015). Probiotic lactic acid bacteria and skin health. Critical Reviews in Food Science and Nutrition, 56, 2331-2337. doi: 10.1080/10408398.2013.834874

Jung, Y, O., Jeong, H., Cho, Y., Lee, E. O., Jang, H. W., Kim, J., Nam, K. T., \& Lim, K. M. (2019). Lysates of a probiotic, Lactobacillus rhamnosus, can improve skin barrier function in a reconstructed human epidermis model. International Journal of Molecular Science, 20, 4289. doi: 10.3390/ijms20174289

Khmaladze, I., Butler, É., Fabre, S., \& Gillbro, J. M. (2019). Lactobacillus reuteri DSM 17938-A comparative study on the effect of probiotics and lysates on human skin. Experimental Dermatology, 28, 822-828. doi: 10.1111/exd.13950

Kim, H., Kim, H. R., Jeong, B. J., Lee, S. S., Kim, T. R., Jeong, J. H., Lee. M., Lee, S., Lee, J. S., \& Chung, D. K. (2015). Effects of oral intake of kimchi-derived Lactobacillus plantarum K8 lysates on skin moisturizing. Journal of Microbiology and Biotechnology, 25, 74-80. doi: 10.4014/jmb.1407.07078

Kim, H. R., Kim, H., Jung, B. J., You, G. E., Jang, S., \& Chung, DK. (2015). Lipoteichoic acid isolated from Lactobacillus plantarum inhibits melanogenesis in B16F10 mouse melanoma cells. Mole- cules and Cells, 38, 163-170. doi: 10.14348/molcells.2015.2263

Kim, K. M., Yang, S. J., Kim, D. S., Lee, C. W., Kim, H. Y., Lee, S., Choi, J. W., Sohn, J., Shin, S. S., \& Lee, S. (2020). Probiotic properties and immune-stimulating effect of the Jeju lava seawater mineral-coated probiotics. LWT-Food Science and Technology, 126, 109299. doi: 10.1016/j.lwt.2020.109299

Kim, N. Y., \& Lee, H. Y. (2015). Enhancement of skin whitening and anti-wrinkle activities of the co-culture of Lactobacillus rhamnosus and Lactobacillus paracasei. Journal of the Society of Cosmetic Scientists of Korea, 41, 253-261. doi: 10.15230/ SCSK.2015.41.3.253

Krutmann, J., Bouloc, A., Sore, G., Bernard, B. A., \& Passeron, T. (2017). The skin aging exposome. Journal of Dermatological Science, 85, 152-161. doi: 10.1016/j.jdermsci.2016.09.015

Lim, H. Y., Jeong, D., Park, S. H., Shin, K. K., Hong, Y. H., Kim, E., Yu, Y. G., Kim, T. R., Kim, H., Lee, J., \& Cho, J. Y. (2020). Antiwrinkle and antimelanogenesis effects of tyndallized Lactobacillus acidophilus KCCM12625P. International Journal of Molecular Sciences, 21, 1620. doi: 10.3390/ijms21051620

Nakamichi, N., Nakao, S., Masuo, Y., Koike, A., Matsumura, N., Nishiyama, M., Al-Shammari, A. H., Sekiguchi, H., Sutoh, K., Usumi, K., \& Kato, Y. (2019). Hydrolyzed salmon milt extract enhances object recognition and location memory through an increase in hippocampal cytidine nucleoside levels in normal mice. Journal of Medicinal Food, 22, 408-415. doi: 10.1089/ jmf.2018.4285

Park, J. H., Lee, M. H., \& Kang, S. M. (2019). The effect of Salix gracilistyla extract on whitening, anti-inflammatory and antiaging. Journal of the Korean Society of Cosmetology, 25, 553562.

Puisina-Ivuc, N. (2008). Skin aging. Acta Dermatovenerologica Alpina, Pannonica et Adriatica, 17, 47-54.

Sasaki, Y., Miyoshi, D., \& Sugimoto, N. (2010). Utilization of salmon milt DNA against UV damage. Applied Biochemistry and Biotechnology, 160, 2458-2466. doi: 10.1007/s12010-009$8697-6$

Sato, A., Kajiya, H., Mori, N., Sato, H., Fukushima, T., Kido, H., \& Ohno, J. (2017). Salmon DNA accelerates bone regeneration by inducing osteoblast migration. PLOS ONE, 12, e0169522. doi: 10.1371/journal.pone.0169522

Shen, X., Nagai, N., Murata, M., Nishimura, D., Sugi, M., \& Munekata, M. (2008). Development of salmon milt DNA/ salmon collagen composite for wound dressing. Journal of Materials Science: Materials in Medicine, 19, 3473-3479. doi: 10.1007/s10856-008-3512-4

Sultana, R., McBain, A. J., \& O’Neill, C. A. (2013). Strain-dependent augmentation of tight-junction barrier function in human primary epidermal keratinocytes by Lactobacillus and Bifidobacterium lysates. Applied and Environmental Microbiology, 79, 4887-4894. doi: 10.1128/AEM.00982-13

You, G. E., Jung, B. J., Kim, H. R., Kim, H. G., Kim, T. R., \& Chung, D. K. (2013). Lactobacillus sakei lipoteichoic acid inhibits MMP-1 induced by UVA in normal dermal fibroblasts of human. Journal of Microbiology and Biotechnology, 23, 13571364. doi: $10.4014 /$ jmb. 1306.06026 